Behavioral/Cognitive

\title{
Relating Visual Production and Recognition of Objects in Human Visual Cortex
}

\author{
DJudith E. Fan, ${ }^{1}$ Jeffrey D. Wammes, ${ }^{2}$ Jordan B. Gunn, ${ }^{3}$ Daniel L.K. Yamins, ${ }^{4}$ CKenneth A. Norman, ${ }^{3}$ \\ and Nicholas B. Turk-Browne ${ }^{2}$ \\ ${ }^{1}$ Department of Psychology, University of California at San Diego, La Jolla, California 92093, 2Department of Psychology, Yale University, New Haven, \\ Connecticut 06520, ${ }^{3}$ Department of Psychology, Princeton University, Princeton, New Jersey 08544, and ${ }^{4}$ Department of Psychology, Stanford University, \\ Stanford, California 94305
}

Drawing is a powerful tool that can be used to convey rich perceptual information about objects in the world. What are the neural mechanisms that enable us to produce a recognizable drawing of an object, and how does this visual production experience influence how this object is represented in the brain? Here we evaluate the hypothesis that producing and recognizing an object recruit a shared neural representation, such that repeatedly drawing the object can enhance its perceptual discriminability in the brain. We scanned human participants $(N=31 ; 11$ male) using fMRI across three phases of a training study: during training, participants repeatedly drew two objects in an alternating sequence on an MR-compatible tablet; before and after training, they viewed these and two other control objects, allowing us to measure the neural representation of each object in visual cortex. We found that: (1) stimulus-evoked representations of objects in visual cortex are recruited during visually cued production of drawings of these objects, even throughout the period when the object cue is no longer present; (2) the object currently being drawn is prioritized in visual cortex during drawing production, while other repeatedly drawn objects are suppressed; and (3) patterns of connectivity between regions in occipital and parietal cortex supported enhanced decoding of the currently drawn object across the training phase, suggesting a potential neural substrate for learning how to transform perceptual representations into representational actions. Together, our study provides novel insight into the functional relationship between visual production and recognition in the brain.

Key words: drawing; fMRI; objects; perception and action; ventral stream

\section{Significance Statement}

Humans can produce simple line drawings that capture rich information about their perceptual experiences. However, the mechanisms that support this behavior are not well understood. Here we investigate how regions in visual cortex participate in the recognition of an object and the production of a drawing of it. We find that these regions carry diagnostic information about an object in a similar format both during recognition and production, and that practice drawing an object enhances transmission of information about it to downstream regions. Together, our study provides novel insight into the functional relationship between visual production and recognition in the brain.

\section{Introduction}

Although visual cognition is often studied by manipulating externally provided visual information, this ignores our ability to

Received July 30, 2019; revised Nov. 23, 2019; accepted Nov. 26, 2019.

Author contributions: J.E.F., D.L.K.Y., K.A.N., and N.B.T.-B. designed research; J.E.F. performed research; J.E.F., J.D.W., and J.B.G. analyzed data; J.E.F. and J.D.W. wrote the first draft of the paper; J.E.F., J.D.W., K.A.N., and N.B.T.-B. edited the paper; J.E.F. and J.D.W. wrote the paper.

This work was supported by National Science Foundation GRFP DGE-0646086 to J.E.F., National Institutes of Health R01 EY021755 and R01 MH069456, and the David A. Gardner ' 69 Magic Project at Princeton University. The code for the analyses presented in this article is available in the following GitHub repository: https://github.com/ cogtoolslab/neurosketch. Links to download the dataset are provided in the documentation for this repository. We thank the Computational Memory and N.B.T.-B. laboratories for helpful comments.

The authors declare no competing financial interests.

Correspondence should be addressed to Judith E. Fan at jefan@ucsd.edu. actively control how we engage with our visual environment. For example, people can select which information to encode by shifting their attention (Chun et al., 2011) and can convey which information was encoded by producing a drawing that highlights this information (Draschkow et al., 2014; Bainbridge et al., 2019). Prior work has provided converging, albeit indirect, evidence that the ability to produce informative visual representations, which we term visual production, recruits general-purpose visual processing mechanisms that are also engaged during visual recognition (James, 2017; Fan et al., 2018). The goal of this paper 


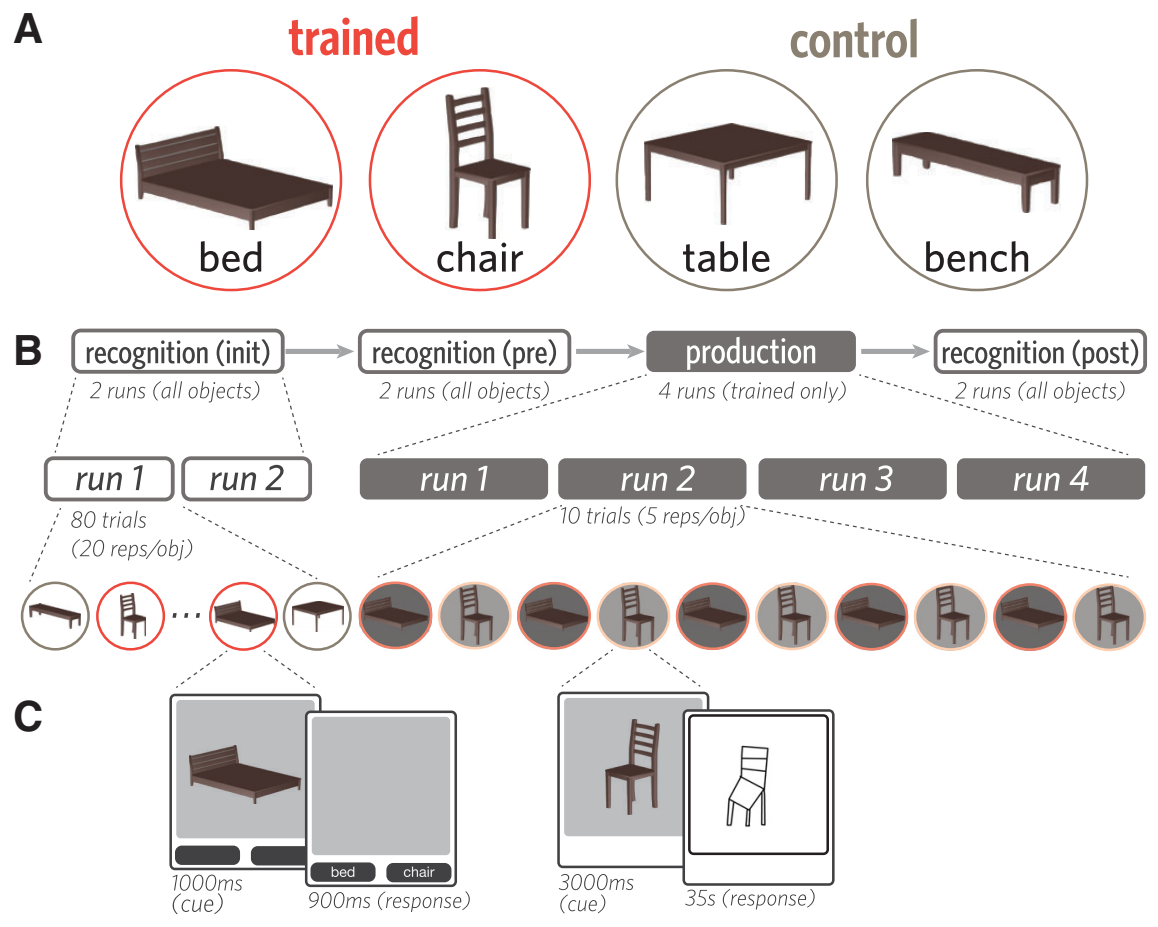

Figure 1. Stimuli, task, and experimental procedure. A, Four 3D objects were used in this study: bed, bench, chair, and table. Each participant was randomly assigned two of these objects to view and draw repeatedly (trained); the remaining two objects were viewed but never drawn (control). B, Before and after the production phase, participants viewed all objects while performing a 2 AFC recognition task. C, On each trial of the recognition phase, one of the four objects was briefly presented ( $1000 \mathrm{~ms})$, followed by a $900 \mathrm{~ms}$ response window. On each trial of the production phase, one trained object was presented ( $3 \mathrm{~s})$, followed by an $35 \mathrm{~s}$ drawing period (i.e., 23 TRs).

is twofold: (1) to more directly characterize the functional role of visual processing mechanisms during visual production; and (2) to investigate how repeated visual production influences neural representations that serve perception and action.

With respect to the first goal, our study builds on prior studies that provided evidence for shared computations supporting visual recognition and visual production. For example, recent work has found that activation patterns in human ventral visual stream measured using fMRI (Walther et al., 2011), as well as activation patterns in higher layers of deep convolutional neural network models of the ventral visual stream (Yamins et al., 2014; Fan et al., 2018), support linear decoding of abstract category information from drawings and color photographs. To what extent are these core visual processing mechanisms also recruited to produce a recognizable drawing of those objects? Initial insights bearing on this question have come from human neuroimaging studies investigating the production of handwritten symbols (though not drawings of real-world objects), revealing general engagement of visual regions during both letter production and recognition (James and Gauthier, 2006; Vinci-Booher et al., 2019). However, the format and content of the representations active in these regions during visual production are not yet well understood.

With respect to the second goal, we build on prior work that has investigated the consequences of repeated visual production. In a recent behavioral study, participants who practiced drawing certain objects produced increasingly recognizable drawings and exhibited enhanced perceptual discrimination of morphs of those objects, suggesting that production practice can refine the object representation used for both production and recognition (Fan et al., 2018). These findings resonate with other evidence that visual production can support learning, including mainte- nance of recently learned information (Wammes et al., 2016) and enhanced recognition of novel symbols (Longcamp et al., 2008; James and Atwood, 2009; Li and James, 2016). Previous fMRI studies that have investigated the neural mechanisms underlying such learning have found enhanced activation in visual cortex when viewing previously practiced letters (James and Gauthier, 2006; James, 2017), and increased connectivity between visual and parietal regions following handwriting experience (Vinci-Booher et al., 2016). However, these studies have focused on univariate measures of BOLD signal amplitude within regions or when analyzing connectivity, raising the question of whether these changes reflect the recruitment of similar representations across tasks or of colocated but functionally distinct representations for each task.

In the current study, we evaluate the hypothesis that producing and recognizing an object recruit a shared neural representation, such that repeatedly drawing the object can enhance its perceptual discriminability in the brain. Our approach advances prior work that has investigated the neural mechanisms underlying production and recognition in two ways: (1) we analyze the pattern of activation across voxels to measure the expression and representation of object-specific information; and (2) we investigate production-related changes to the organization of object representations, specifically changes in patterns of voxelwise connectivity among ventral and dorsal visual regions as a consequence of production practice.

\section{Materials and Methods}

\section{Participants}

Based on initial piloting, we developed a target sample size of 36 human participants, across whom all condition and object assignments would be fully counterbalanced. Participants were recruited from the Princeton, New Jersey community, were right-handed, and provided informed consent in accordance with the Princeton Institutional Review Board. Of the 39 participants who were recruited, 33 participants successfully completed the session. After accounting for technical issues during data acquisition (e.g., excessive head motion), data from 31 participants (11 male, 23.2 years) were retained.

\section{Stimuli}

Four objects from the furniture category were used in this study, based on a prior study (Fan et al., 2018): bed, bench, chair, and table. These objects were represented by 3D mesh models constructed in Autodesk Maya to contain the same number of vertices and the same brown surface texture, and thereby share similar visual properties apart than their shape (Fig. $1 A$ ). Each of these objects was rendered from a $10^{\circ}$ viewing angle (i.e., slightly above) at a fixed distance on a gray background in 40 viewpoints (i.e., each rotated by an additional $9^{\circ}$ about the vertical axis).

\section{Experimental design}

Each participant was randomly assigned two of the four objects to practice drawing repeatedly ("trained" objects). The remaining two objects ("control" objects) provided a baseline measure of changes in neural representations in the absence of drawing practice. At the beginning of each session and outside of the scanner, participants were familiarized 
with each of the four objects while being briefed on the overall experimental procedure. There were four phases in each session (Fig. $1 B, C$ ), all of which were scanned with fMRI: initial recognition (two runs), prepractice recognition (two runs), production practice (four runs), and a postpractice recognition phase (two runs).

\section{Recognition task}

Within each of the three recognition phases, participants viewed all four objects in all 40 viewpoints once each and performed an object identification cover task. Repetitions of each object were divided evenly across the two runs of each phase, and in a random order within each run, interleaved with other objects. On each recognition trial, participants were first presented with one of the objects $(1000 \mathrm{~ms})$. The object then disappeared, and two labels appeared below the image frame, one of which corresponded to the correct object label. Participants then made a speeded forced-choice judgment about which of the two objects they saw by pressing one of two buttons corresponding to each label within a 900 ms response window. The assignment of labels to buttons was randomized across trials. Participants did not receive accuracy-related feedback but received visual feedback if their response was successfully recorded within the response window (selected button highlighted). Interstimulus intervals were jittered from trial to trial by sampling from the following durations, which appeared in a fixed proportion in each run to ensure equal run lengths: $3000 \mathrm{~ms}$ interstimulus interval ( $40 \%$ trials/run), 4500 $\mathrm{ms}(40 \%), 6000 \mathrm{~ms}(20 \%)$. Each run was $6 \mathrm{~min}$ in length, and no object appeared in the first or final $12 \mathrm{~s}$ of each run.

\section{Production task}

Participants produced drawings on a pressure-sensitive MR-compatible drawing tablet (Hybridmojo) positioned on their lap by using an MRcompatible stylus, which they held like a pencil in the right hand. Before the first drawing run, participants were familiarized with the drawing interface. They practiced producing several closed curves approximately the size of the drawing canvas, to calibrate the magnitude of drawing movements on the tablet (which they could not directly view) to the length of strokes on the canvas. They also practiced drawing two other objects of their choice, providing them with experience drawing more complex shapes using this interface. When participants did not spontaneously generate their own objects to draw, they were prompted to draw a house and a bicycle.

In each of the four runs of the production phase, participants drew both trained objects 5 times each in an alternating order, producing a total of 20 drawings of each object. Each production practice trial had a fixed length of $45 \mathrm{~s}$. First, participants were cued with one of the trained objects (3000 ms). Following cue offset and a 1000 ms delay, a blank drawing canvas of the same dimensions appeared in the same location. We refer to the trained object currently being drawn as the target object, and to the other trained object not currently being drawn as the foil object. Participants then used the subsequent $35 \mathrm{~s}$ to produce a drawing of the object before the drawing was automatically submitted. Following drawing submission, the canvas was cleared, and there was a $6000 \mathrm{~ms}$ delay until the presentation of the next object cue. Participants were cued with 20 distinct viewpoints of each trained object in a random sequence ( $18^{\circ}$ rotation between neighboring viewpoints), were instructed to draw each target object in the same orientation as in the image cue, and did not receive performance-related feedback. Each run was $7.7 \mathrm{~min}$ in length and contained rest periods during the first $12 \mathrm{~s}$ and final $45 \mathrm{~s}$ of each run.

\section{Statistics}

We primarily used nonparametric analysis techniques (i.e., bootstrap resampling) to estimate parameters of interest (Efron and Tibshirani, 1994), and provide 95\% CIs for these parameter estimates. We favored this approach due to its emphasis on estimation of effect sizes, by contrast with the dichotomous inferences yielded by traditional null-hypothesis significance tests (Cumming, 2014). Furthermore, all applications of logistic regression-based classification on $\mathrm{fMRI}$ data to derive these parameter estimates were conducted in a cross-validated manner. Changes in classifier output over time were fit with linear mixed-effects regression models (Bates et al., 2015), which included random intercepts for different participants.

\section{fMRI data acquisition}

All fMRI data were collected on a 3T Siemens Skyra scanner with a 64-channel head coil. Functional images were obtained with a multiband EPI sequence $\left(\mathrm{TR}=1500 \mathrm{~ms}, \mathrm{TE}=30 \mathrm{~ms}\right.$, flip angle $=70^{\circ}$, acceleration factor $=4$, voxel size $=2 \mathrm{~mm}$ isotropic), yielding 72 axial slices that provided whole-brain coverage. High-resolution T1-weighted anatomical images were acquired with an MPRAGE sequence $(\mathrm{TR}=2530 \mathrm{~ms}$, $\mathrm{TE}=3.30 \mathrm{~ms}$, voxel size $=1 \mathrm{~mm}$ isotropic, 176 slices, $7^{\circ}$ flip angle).

\section{fMRI data preprocessing}

fMRI data were preprocessed with FSL (http://fsl.fmrib.ox.ac.uk). Functional volumes were corrected for slice acquisition time and head motion, high-pass filtered (100 s period cutoff), and aligned to the middle volume within each run. For each participant, these individual runaligned functional volumes were then registered to the anatomical T1 image, using boundary-based registration. All participant-level analyses were performed in participants' own native anatomical space. For grouplevel analyses and visualizations, functional volumes were projected into MNI standard space.

\section{fMRI data analysis}

Head motion. Given the distal wrist/hand motion required to produce drawings, it was important to measure and verify that there was not extreme head motion during drawing production relative to rest periods (i.e., cue presentation, and delay). For each production run, the time courses for estimated rotations, translations, and absolute/relative displacements were extracted from the output of MCFLIRT. Functional data were partitioned into production (i.e., the 23 TRs spent drawing in each TR) and rest (i.e., during cue presentation or delay between trials) volumes. We found that there was no difference in rotational movement between production and rest periods (mean $=-0.0001 ; 95 \%$ CI $=$ $[-0.0003,0.0001])$. Indeed, there was reliably less head movement during production relative to rest, as measured by translation (mean = $-0.006 ; 95 \% \mathrm{CI}=[-0.011,-0.002])$, absolute (mean $=-0.027 ; 95 \%$ $\mathrm{CI}=[-0.054,-0.004])$, and relative displacement $($ mean $=-0.016$; $95 \% \mathrm{CI}=[-0.024,-0.008])$.

Defining ROIs in occipitotemporal cortex. We focused our analyses on nine ROIs in occipitotemporal cortex: V1, V2, lateral occipital cortex (LOC), fusiform (FUS), inferior temporal lobe (IT), parahippocampal cortex (PHC), perirhinal cortex (PRC), entorhinal cortex (EC), and hippocampus (HC). These regions were selected based on prior evidence for their functional involvement in visual processing. For instance, neurons in V1 and V2 are tuned to the orientation of perceived contours, which constitute simple line drawings and also often define the edges of an object (Hubel and Wiesel, 1968; Gegenfurtner et al., 1996; Kamitani and Tong, 2005; Sayim and Cavanagh, 2011). Likewise, neural populations in higher-level ventral regions, including LOC, FUS, and IT, have been shown to play an important role in representing more abstract invariant properties of objects (Gross, 1992; Grill-Spector et al., 2001; Kourtzi and Kanwisher, 2001; Hung et al., 2005; Rust and DiCarlo, 2010), with medial temporal regions including $\mathrm{PHC}, \mathrm{PRC}, \mathrm{EC}$, and $\mathrm{HC}$ participating in both online visual processing, as well as the formation of visual memories (Murray and Bussey, 1999; Epstein et al., 2003; Davachi, 2006; Schapiro et al., 2012; Garvert et al., 2017). Masks for each ROI were defined in each participants' T1 anatomical scan, using FreeSurfer segmentations (http://surfer.nmr.mgh.harvard.edu/).

Defining production-related regions in parietal cortex and precentral gyrus. Motivated by prior work investigating visually guided action (Goodale and Milner, 1992; Vinci-Booher et al., 2019), we also sought to analyze how sensory information represented in occipital cortex is related to downstream regions associated with action planning and execution, including parietal and motor cortex. Accordingly, ROI masks for parietal cortex and precentral gyrus were also generated for each participant based on their Freesurfer segmentation. To determine which voxels across the whole brain were specifically engaged during production, a group-level univariate activation map was estimated, contrasting production versus rest. To derive these production task-related activation 


\section{A EXtract VOXEl ACtivation PATterns to EACH OBJECT WITHIN EACH ROI}
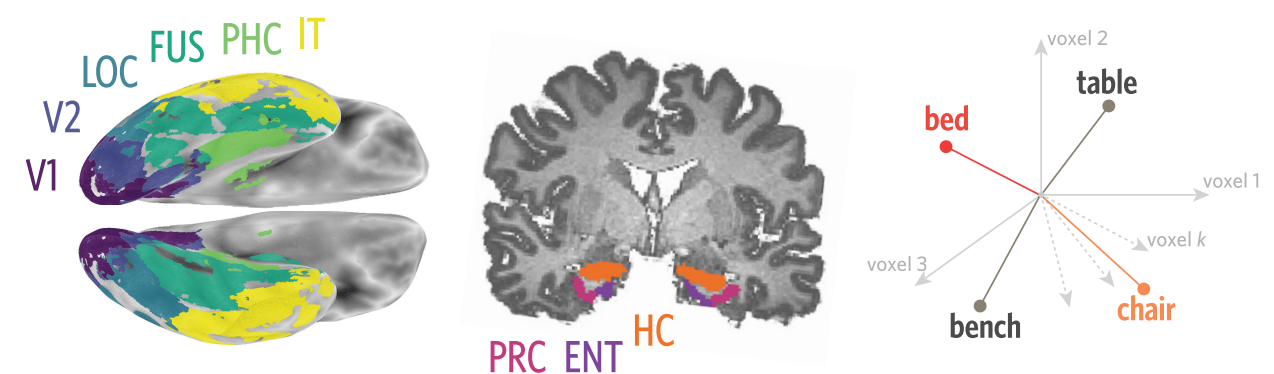

\section{B TRAIN CLASSIFIER ON RECOGNITION DATA}

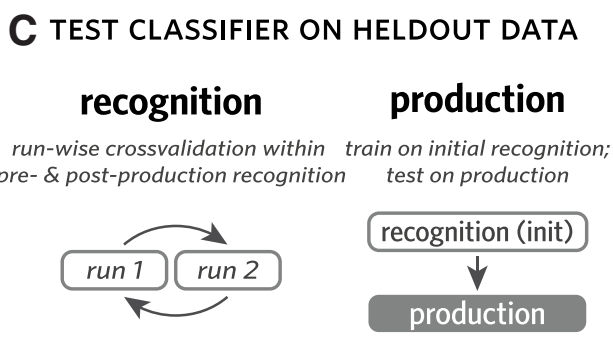

Figure 2. Measuring object evidence in activation patterns during recognition and production. $\boldsymbol{A}$, For each participant, anatomical ROls were defined using FreeSurfer. Activation patterns across voxels in each ROI were extracted for each recognition trial and for all time points of each production trial. These activation patterns can be expressed as vectors in a $k$-dimensional vector space, where k reflects the number of voxels in a given ROI. $\boldsymbol{B}$, Evidence for each object was measured using a 4-way logistic regression classifier trained on activation patterns from recognition runs to predict the current object being viewed or drawn (e.g., bed), and discriminate it from the other three objects (i.e., bench, chair, table). This classifier can be used to measure both the general expression of object-specific information, measured by classification accuracy, as well as the degree of evidence for particular objects, measured by the probabilities it assigns to each. C, To measure object evidence during recognition, this classifier was trained in a runwise cross-validated manner within each of the preproduction and postproduction phases. To measure object evidence during production, the same type of classifier was trained on data from the initial recognition phase.

maps, we analyzed each production run with a GLM. Regressors were specified for each trained object by convolving a boxcar function, reflecting the total amount of time spent drawing (i.e., 23 TRs, or $34.5 \mathrm{~s}$ ), with a double-gamma hemodynamic response function (HRF). A univariate contrast was then applied, with equal weighting on the regressors for each trained object, to determine the clusters of voxels that were preferentially active during drawing production, relative to rest. Voxels that exceeded a strict threshold $(Z=3.1)$ and also lay within the anatomically defined ROI boundaries (i.e., in occipital cortex, parietal cortex, or precentral gyrus) were included.

To avoid statistical dependence between this procedure used for voxel selection and for subsequent classifier-based analyses, we defined participant-specific activation maps in a leave-one-participant-out fashion. That is, a held-out participant's production mask was constructed based solely on the basis of task-related activations from all remaining participants. Once each participant's mask was defined, we took the intersection between this map and the participant's own anatomically defined cortical segmentation to construct the production-related ROIs in V1, V2, LOC, parietal cortex, and precentral gyrus). We had no a priori predictions about hemispheric differences, so ROI masks were collapsed over the left and right hemispheres.

Measuring object evidence during recognition and production phases. To quantify the expression of object-specific information throughout recognition and production, we analyzed the neural activation patterns across voxels associated with each object (Haxby et al., 2001; Kamitani and Tong, 2005; Norman et al., 2006; Cohen et al., 2017). Specifically, we extracted neural activation patterns evoked by each object cue during recognition, measured 3 TRs following each stimulus offset to account for hemodynamic lag. We used these patterns to train a 4-way logistic regression classifier with $\mathrm{L} 2$ regularization to predict the identity of the current object in either held-out recognition data or production data. This procedure was performed separately in each ROI in each participant, and all raw neural activation patterns were $z$-scored within voxel and within run before be used for either classifier training or evaluation.
To measure object evidence during recognition, we applied the classifier in a twofold cross-validated fashion within each of the preproduction and postproduction phases, such that, for each fold, the data from one run were used as training whereas the data from the other run were used for evaluation. Aggregating predictions across folds, we computed the proportion of recognition trials on which the classifier correctly identified the currently viewed object, providing a benchmark estimate of how much object-specific information was available from neural activation patterns during recognition. We constructed 95\% CIs for estimates of decoding accuracy for each ROI by bootstrap resampling participants 10,000 times.

To measure object evidence during production, we trained the same type of classifier exclusively on data from the initial recognition phase, which minimized statistical dependence on the classifier based on preproduction and postproduction phases. We then evaluated this classifier on every time point while participants produced their drawings, which consisted of the 23 TRs following the offset of the image cue, shifted forward 3 TRs to account for hemodynamic lag (Fig. 2).

Because this type of classifier assigns a probability value to each object, it can be used to evaluate the strength of evidence for each object at each time point. To evaluate the degree to which the currently drawn object (target) was prioritized, we extracted the classifier probabilities assigned to the target, foil, and two control objects on each TR during drawing production. We then used these probabilities to derive metrics that quantify the relative evidence for one object compared with the others. Specifically, we define "target selection" as the log-odds ratio between the target and foil objects $(\operatorname{In}[p($ target $) / p($ foil $)])$, which captures the degree to which the voxel pattern is more diagnostic of the target than the foil. We define "target evidence" as the log-odds ratio between the target and the mean natural-log probabilities assigned to the two control objects for each time point, which captures the degree to which the voxel pattern is more diagnostic of the target than the baseline control objects. We likewise define "foil evidence" as the log-odds ratio between the foil object and the mean natural log probabilities for the 2 control objects, which captures the degree to which the voxel pattern is more diagnostic of the 


\section{ANALYZE VOXEL CONNECTIVITY PATTERNS BETWEEN ROIS DURING PRODUCTION}

\section{A voxel selection}

\section{intersection between functional and anatomical maps}
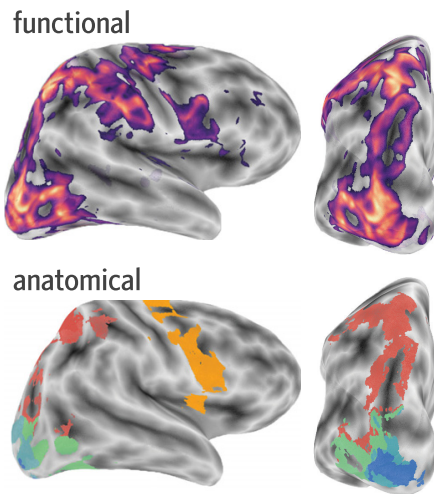

production

vs. rest

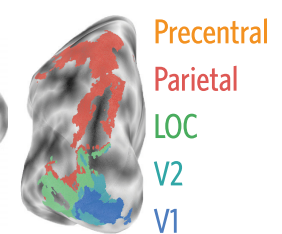

\section{B connectivity pattern}

temporal correlation btw all voxels in one region \& all voxels in second region

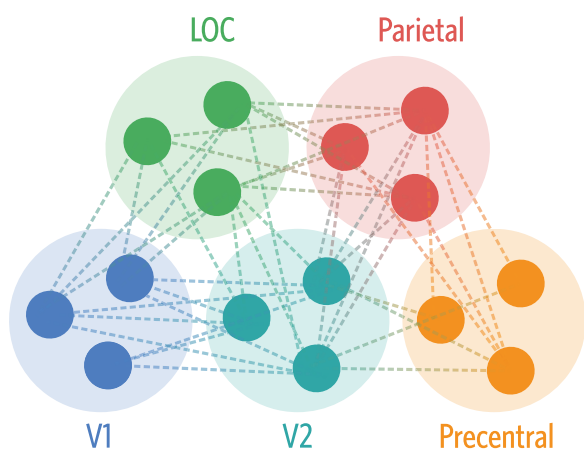

\section{C construct classifier}

crossvalidated object classifier on connectivity patterns

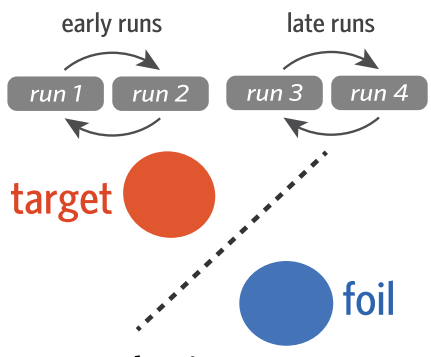

\section{D target selection}

relative evidence for target vs. foil

$\log$ odds $=\log \left[\frac{p(\text { target })}{p(\text { foil })}\right]$

Figure 3. Measuring object evidence in connectivity patterns between regions during production. $A$, Voxels in each of several anatomical ROIs (i.e., V1, V2, LOC, parietal, precentral) that were also consistently engaged during the production task were included in this analysis. To determine which voxels were consistently engaged during production, while minimizing statistical dependence between voxel selection and multivoxel pattern analysis, a production task-related activation map was generated in a leave-one-participant-out manner. $\boldsymbol{B}$, Connectivity patterns were computed for each trial, for each pair of ROls. Each connectivity pattern consists of the set of $m \times n$ pairwise temporal correlations between every voxel in one ROI (containing $m$ voxels) with every voxel in the second ROI (containing $n$ voxels). The temporal correlation between each pair of voxels reflects the correlation between the activation time series for the first voxel and the activation time series for the second voxel, over all 23 TRs in each production trial. C, Connectivity patterns were used to construct a 2-way logistic regression classifier to discriminate the currently drawn object (target) from the other trained object (foil). This classifier was trained in a runwise cross-validated manner within the first two runs (early) and the final two runs (late) of the production phase. $\boldsymbol{D}$, Target selection, the degree to which the target was prioritized over the foil, was defined as the log-odds ratio between the target and foil objects.

foil than the baseline control objects. For each ROI within a participant, we compute the average target selection, target evidence, and foil evidence across time points in all four production runs, then aggregate these estimates across participants to compute a group-level estimate for each metric and CI derived via bootstrap resampling of participants 1000 times.

Measuring object evidence in connectivity patterns during production phase. The above approach to analyzing multivariate neural representations during production focuses on spatially distributed activation patterns within individual regions in visual cortex. However, visual production inherently entails not only recruitment of individual regions, but also coordination between them. Practice producing drawings of an object may lead to changes in how information is shared between early sensory regions and downstream visuomotor regions. Such changes may reflect different ways that information in the visual representation of the object cue may be selected or transformed to guide action selection during drawing production. Because such coordination inherently involves multiple brain regions, we did not expect that it would be directly available in activation patterns within any given region. Accordingly, we developed an approach to explore how object-specific information might be shared between regions during drawing production. Specifically, because prior work has indicated that parietal and motor regions are also recruited during visual production (Vinci-Booher et al., 2019), we measured how activation patterns in visual cortex are related to activation patterns in these regions during drawing production.

For each pair of ROIs (e.g., V1 and parietal), we extracted the connectivity pattern from every production trial (Fig. 3). Each connectivity pattern consists of the $m \times n$ pairwise temporal correlations between every voxel in one ROI (containing $m$ voxels) with every voxel in the second ROI (containing $n$ voxels). The temporal correlation between each pair of voxels reflects the correlation between the activation time series for the first voxel and the activation time series for the second voxel, over all 23 TRs in each production trial.

For each pair of ROIs, we then trained a 2-way logistic regression classifier to discriminate the target versus foil objects based on these connectivity patterns. The classifier was trained in a runwise cross- validated manner within the first two runs (early) and the final two runs (late) of the production phase. To capture the degree to which the connectivity pattern was more diagnostic of the target than the foil, we computed target selection, which was averaged over all trials within a phase (early or late). With this approach, we computed connectivity over time within a trial, treating the connectivity matrix from each trial as an individual observation that was used to train the classifier, then compared the success of the classifier on other trials from the same half of the production phase to determine whether target selection increased from the first half to the second half.

Data were fit with a linear mixed-effects regression model (Bates et al., 2015) that included time (early vs late) as a predictor and random intercepts for different participants. We compared this model with a baseline model that did not include time as a predictor. The reliability of the increase in target selection across time was measured in two ways: (1) formal model comparison to evaluate the extent to which including time as a predictor improved model fit; and (2) the construction of bootstrapped $95 \%$ CIs for estimates of the effect of time to evaluate whether they spanned zero (or chance). To further evaluate whether connectivity patterns carried task-related information that was not redundant with the activation patterns within regions, we conducted a control analysis, which involved constructing the same type of classifier on voxel activation patterns extracted from two ROIs at a time, rather than the pattern of connectivity between them.

\section{Results}

Discriminable object representations in visual cortex during recognition

Following prior work (Haxby et al., 2001; Norman et al., 2006; Cichy et al., 2011; Cohen et al., 2017), we hypothesized that there would be consistent information about the identity of each object in visual cortex across repeated presentations during the recognition phase. Specifically, we predicted that the stimulus-evoked pattern of neural activity across voxels in visual cortex upon viewing an object could be used to reliably decode its identity. To test 


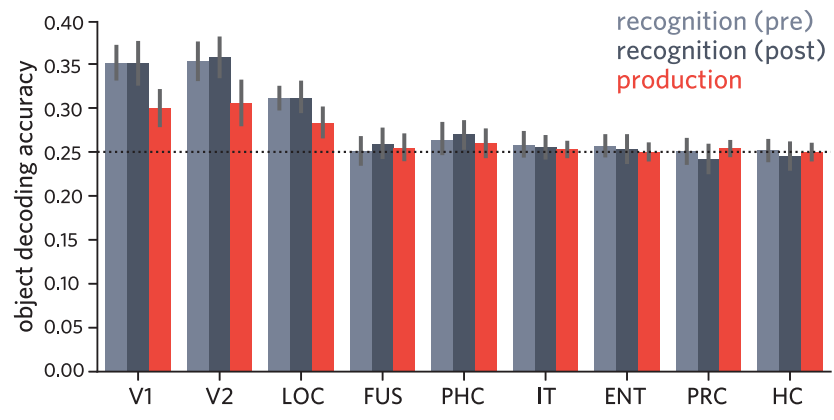

Figure 4. Accuracy of object classifier during pre/post recognition phase and drawing production phase, for each ventral visual R0I. Error bars indicate $95 \% \mathrm{Cls}$.

this prediction, we first extracted neural activation patterns evoked by each object during recognition separately for each participant, in each occipitotemporal ROI. We used neural activation patterns extracted from a subset of recognition-phase data to train a 4-way logistic regression classifier that could be used to evaluate decoding accuracy on held-out recognition data in the same regions (Fig. 2). We computed a twofold cross-validated measure of object decoding accuracy (Fig. 4), wherein for each of the preproduction and postproduction phases, the 40 repetitions from one of the two runs were used for training the classifier, whereas the 40 repetitions from the other run were used for evaluation.

We found that the identity of the currently viewed object could be reliably decoded in V1, V2, and LOC in the preproduction recognition phase $(95 \%$ CIs: $\mathrm{V} 1=[0.332,0.370], \mathrm{V} 2=$ $[0.332,0.374]$, LOC $=[0.299,0.324]$; chance $=0.25$; Fig. 4$)$, but not in the more anterior ROIs (95\% CIs: FUS $=[0.236,0.266]$, $\mathrm{PHC}=[0.248,0.280], \mathrm{IT}=[0.245,0.272], \mathrm{ENT}=[0.246$, $0.268], \mathrm{PRC}=[0.237,0.264], \mathrm{HC}=[0.241,0.263])$. Likewise, we found that the same early visual regions, as well as PHC, supported above-chance decoding during the postproduction phase (95\% CIs: V1 $=[0.327,0.374], \mathrm{V} 2=[0.337,0.379]$, LOC $=$ $[0.296,0.329], \mathrm{PHC}=[0.255,0.286])$, but not the other regions $(95 \%$ CIs: FUS $=[0.244,0.275]$, IT $=[0.242,0.268]$, ENT $=$ $[0.238,0.268], \mathrm{PRC}=[0.227,0.258], \mathrm{HC}=[0.232,0.259])$. These results suggest that information about object identity was not uniformly accessible from all regions along the ventral stream, but primarily in occipital cortex, consistent with previous work (Grill-Spector et al., 2001; Güclü and van Gerven, 2015).

\section{Similar object representations in visual cortex during recognition and production}

The results so far show that there is robust object-specific information evoked by visual recognition of each object in the patterns of neural activity in V1, V2, and LOC. Based on prior work (Fan et al., 2018), we further hypothesized that the neural object representation evoked during recognition would be functionally similar to that recruited during drawing production. Specifically, we predicted that consistency in the patterns of neural activity evoked in visual cortex upon viewing an object could be leveraged to decode the identity of that object during drawing production, even during the period when the object cue was no longer visible. To test this prediction, we evaluated how well a linear classifier trained exclusively on recognition data to decode object identity could generalize to production data in the same regions.

For each ROI in each participant, we used activation patterns evoked by each object across 40 repetitions in two initial recognition runs to train a 4 -way logistic regression classifier, which we then applied to each time point across the four production practice runs. Critically, we restricted our classifier-based evaluation of production data to the 23 TRs following the offset of the object cue in each trial, providing a measure of the degree to which object-specific information was available in each ROI during production throughout the period when the object was no longer visible. Moreover, we ensured that the data used to train this classifier came from different runs than those used to measure the expression of object-specific information in these regions during the preproduction and postproduction recognition phases. Averaging over all TRs during production, we found reliable decoding of object identity in V1 (mean $=0.3 ; 95 \% \mathrm{CI}=[0.280$, $0.320]$, chance $=0.25$; Fig. 4$)$, V2 (mean $=0.305 ; 95 \% \mathrm{CI}=$ $[0.281,0.331])$, and LOC (mean $=0.283 ; 95 \% \mathrm{CI}=[0.267$, $0.299]$ ), though not in the more anterior ROIs (95\% CIs: FUS = $[0.241,0.268], \mathrm{PHC}=[0.244,0.275], \mathrm{IT}=[0.245,0.261], \mathrm{EC}=$ $[0.241,0.259], \mathrm{PRC}=[0.246,0.262], \mathrm{HC}=[0.241,0.258]$; Fig. 4).

These results suggest that, despite large differences between the two tasks, that is, visual discrimination of a realistic rendering versus production of a simple sketch based on object information in working memory, there are functional similarities between the visually evoked representation of objects in occipital cortex (i.e., $\mathrm{V} 1, \mathrm{~V} 2, \mathrm{LOC}$ ) and the representation that is recruited during the production of drawings of these objects.

\section{Sustained selection of target object during production in visual cortex}

The findings so far show that the identity of the currently drawn object can be linearly decoded from voxel activation patterns in occipital cortex during drawing production. While this speaks to the overall prioritization of the currently drawn target object in visual cortex, it is unclear whether this prioritization is specific to the target. On the one hand, it may be that both trained objects were activated to a similar and heightened degree during the production phase relative to the control objects because participants alternated between these objects. On the other hand, this alternation may have led participants to selectively prioritize the target object, resulting in the foil object not only being less activated than the target, but also suppressed relative to the control objects. To tease these possibilities apart, we quantified the relative evidence for each object on every time point during drawing production, in each ventral stream ROI (Fig. 5).

We found sustained target evidence (target $>$ control) across the production phase in $\mathrm{V} 1$ (mean $=0.228 ; 95 \% \mathrm{CI}=[0.102$, $0.361]), \mathrm{V} 2($ mean $=0.227 ; 95 \% \mathrm{CI}=[0.094,0.360])$, and LOC $($ mean $=0.128 ; 95 \% \mathrm{CI}=[0.035,0.231])$, consistent with the classifier accuracy results reported above. We did not find reliable evidence for sustained target evidence in the other ROIs (95\% CIs: FUS $=[-0.025,0.222], \mathrm{PHC}=[-0.067,0.056], \mathrm{IT}=$ $[-0.163,0.026], \mathrm{EC}=[-0.113,0.020], \mathrm{PRC}=[-0.103,0.018]$, $\mathrm{HC}=[-0.047,0.062])$.

We also found reliable negative foil evidence (foil $<$ control) across the production phase again in $\mathrm{V} 1$ (mean $=-0.449 ; 95 \%$ $\mathrm{CI}=[-0.601,-0.295]), \mathrm{V} 2($ mean $=-0.481 ; 95 \% \mathrm{CI}=[-0.701$, $-0.261]$ ), and LOC (mean $=-0.188 ; 95 \% \mathrm{CI}=[-0.277$, $-0.095])$, suggesting that not only is the task-relevant target object prioritized in these regions, but also that the presently taskirrelevant foil object is suppressed. Again, we did not find reliable evidence for sustained foil evidence (in either direction) in the other ROIs (95\% CIs: FUS $=[-0.170,0.067], \mathrm{PHC}=[-0.030$, $0.072], \mathrm{IT}=[-0.154,0.06], \mathrm{EC}=[-0.119,0.013], \mathrm{PRC}=$ $[-0.05,0.056], \mathrm{HC}=[-0.064,0.051])$. 


\section{A OBJECT EVIDENCE ACROSS REPETITIONS DURING PRODUCTION PHASE}
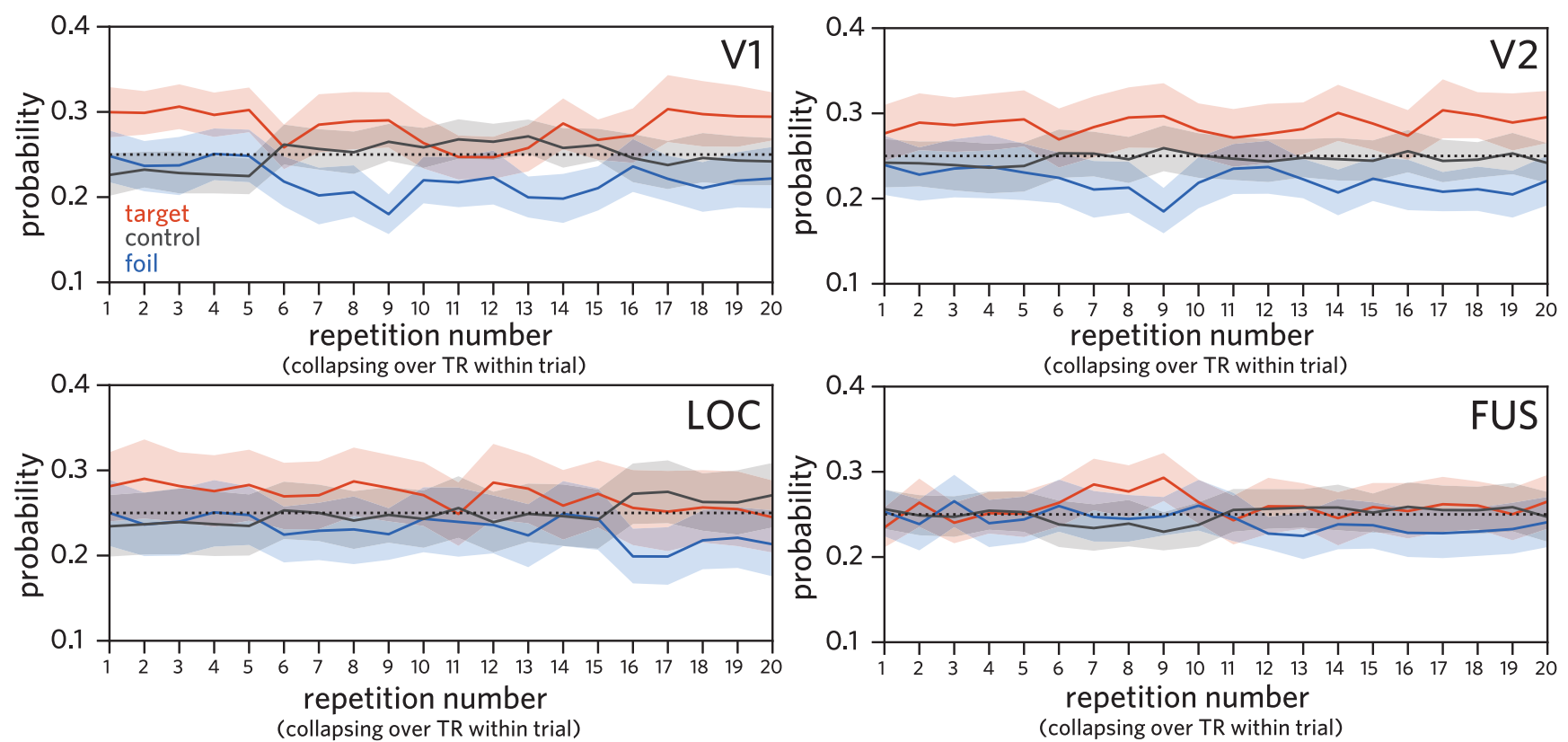

B OBJECT EVIDENCE ACROSS TIME POINTS WITHIN PRODUCTION TRIAL
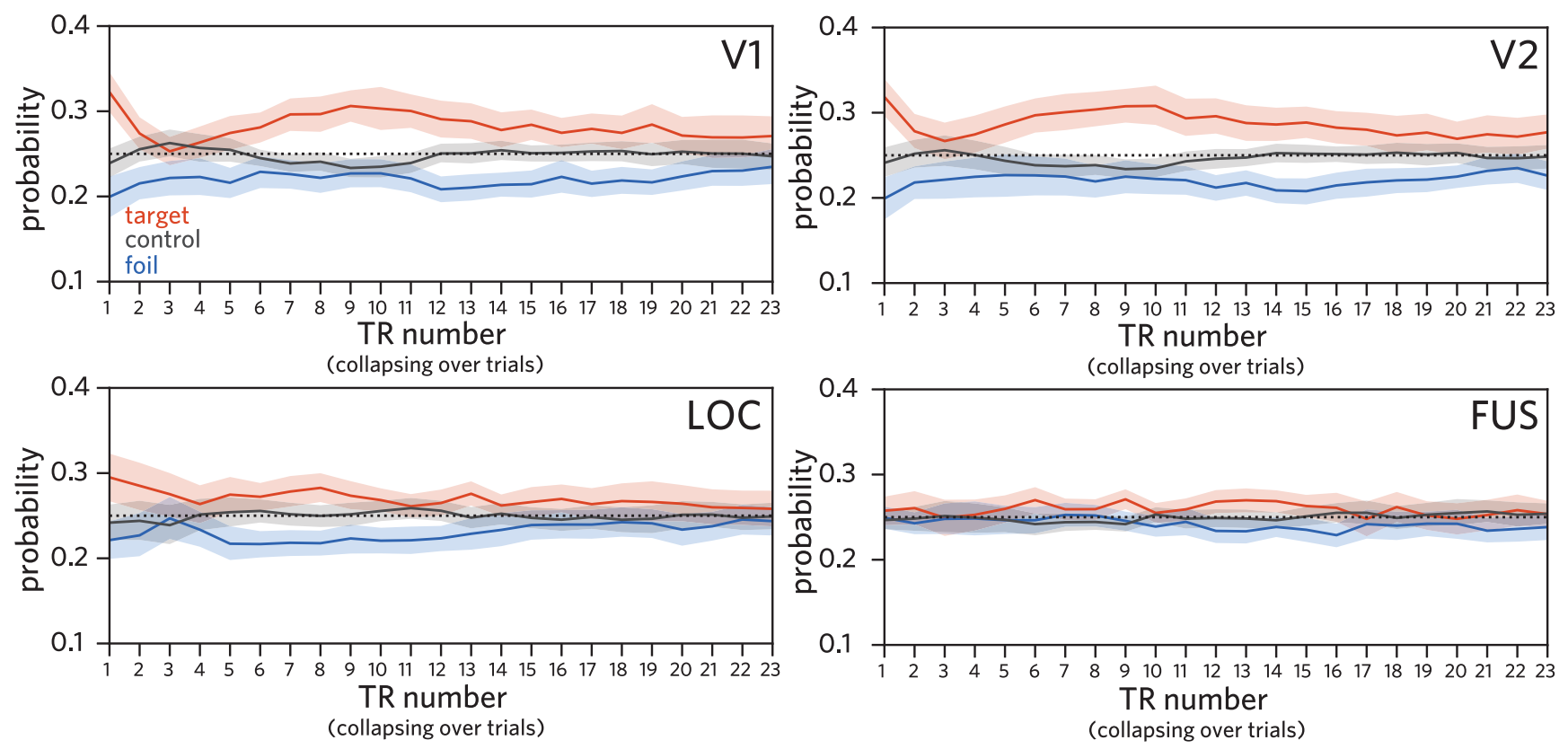

Figure 5. Classifier evidence for each object over time during production, trained on recognition activation patterns. $\boldsymbol{A}$, Classifier evidence for target (currently drawn), foil (other trained), and control (never drawn) objects across repetitions during production phase in V1, V2, LOC, and FUS, averaging over TR within trial. $\boldsymbol{B}$, Classifier evidence for each object by TR within trial in the same regions, averaging over trials. Probabilities assigned by a 4-way logistic regression classifier trained on patterns of neural responses evoked during initial recognition of these objects. Shaded regions represent $95 \%$ confidence bands.

Finally, we found sustained target selection (target $>$ foil) across the production phase again in V1 (mean $=0.676 ; 95 \%$ $\mathrm{CI}=[0.449,0.906]), \mathrm{V} 2($ mean $=0.708 ; 95 \% \mathrm{CI}=[0.484$, $0.955])$, LOC $($ mean $=0.316 ; 95 \% \mathrm{CI}=[0.216,0.423])$, and additionally in FUS (mean $=0.151 ; 95 \% \mathrm{CI}=[0.074,0.229]$ ). Again, we did not find reliable evidence for sustained target selection in the other ventral stream ROIs $(95 \%$ CIs: PHC $=$ $[-0.081,0.0262], \mathrm{IT}=[-0.098,0.056], \mathrm{EC}=[-0.053,0.063]$, $\mathrm{PRC}=[-0.112,0.022], \mathrm{HC}=[-0.041,0.068])$.
Overall, these results show that the currently drawn object was selectively prioritized in occipital cortex, relative to both neverdrawn control objects and the other trained object, which was reliably suppressed below the control-object baseline throughout the production phase.

A related question raised by these findings concerns the degree to which object decodability during drawing production was driven by active recruitment of an internal object representation or by sensory exposure to the finished drawing. Our current data 
provide some support for the contribution of both sources: First, we observe reliable target evidence and negative foil evidence both at early time points and throughout each production trial, especially in regions where target selection is most pronounced (i.e., V1 and V2). Such sustained selection is suggestive of an active internal prioritization of the target object, accompanied by suppression of the foil object (Fig. 5B). Second, we observe a steady increase in target evidence throughout the period of each production trial when participants were most actively engaged in drawing. It is during this period that participants were exposed to increasing sensory evidence for the target object, provided by the increasingly recognizable drawing they were producing.

Together, these findings suggest the operation of a selection mechanism during drawing production that simultaneously enhances the currently relevant target object representation and suppresses the currently irrelevant foil object representation in early visual cortex, potentially involving mechanisms similar to those supporting selective attention and working memory (Tipper et al., 1994; Serences et al., 2009; Gazzaley and Nobre, 2012; Lewis-Peacock and Postle, 2012; Fan and Turk-Browne, 2013).

\section{Stable object representations in activation patterns in visual cortex}

Because we collected neural responses to each object both before and after the production phase, we could also evaluate the consequences of repeatedly drawing an object on the discriminability of neural activation patterns associated with each object in these regions. Insofar as repeatedly drawing the trained objects led to more discriminable representations of those objects within each region, we hypothesized that trained object representations would become more differentiated following training, resulting in enhanced object decoding accuracy in the postproduction phase relative to the preproduction phase, especially for trained objects. To test this hypothesis, we analyzed changes using a linear mixed-effects model with phase (pre vs post) and condition (trained vs control) as predictors of decoding accuracy, with random intercepts for each participant. We did not find evidence that objects differed in discriminability between the preproduction and postproduction recognition phases in any ROI (i.e., no main effect of phase; $p$ values $>0.225$ ), nor evidence for larger changes in discriminability for trained versus control objects (i.e., no phase $\times$ condition interaction, $p$ values $>0.135$ ). These results suggest that stimulus-evoked neural activation patterns in occipital cortex were stable under the current manipulation of visual production experience.

\section{Enhanced object evidence in connectivity patterns across occipitotemporal and parietal regions}

Drawing is a complex visuomotor behavior, involving the concurrent recruitment of occipitotemporal cortex, as well as downstream parietal and motor regions (Vinci-Booher et al., 2019). In agreement with prior work, we found consistent engagement in voxels within V1, V2, LOC, parietal cortex, and precentral gyrus during drawing production relative to rest, as measured by a univariate contrast (see Materials and Methods). We asked whether this joint engagement may reflect, at least in part, the transmission of object-specific information among these regions. If so, then learning to draw an object across repeated attempts may lead to enhanced transmission of the diagnostic features of the object. To explore whether there was enhanced transmission of objectspecific information, we investigated connectivity patterns between voxels in V1, V2, LOC, parietal cortex, and precentral gyrus engaged during the production task.
Specifically, using the entire 23 TR time course of a given trial, we computed a voxelwise connectivity matrix for each pair of these ROIs. Each trial's connectivity matrix was then used as input to a binary logistic regression classifier, trained with L2 regularization to predict object identity separately in each half of the production phase. For each test trial, the classifier yielded two probability values corresponding to the amount of evidence for the target and foil objects. As in the previous analysis, we computed a target selection log-odds ratio, this time for each test trial and for each participant in every pair of ROIs. The trials were then divided based on whether they were early (runs 1 and 2) or late (runs 3 and 4) in the production phase. We then analyzed changes in target selection as a function of half using a linear mixed-effects model with random intercepts for each participant. This analysis revealed the extent to which patterns of connectivity between regions during each drawing trial became more diagnostic of object identity over time.

When analyzing changes in connectivity patterns between $\mathrm{V} 1$ and V2, we found that including time as a predictor improved model fit $\left(\chi_{(1)}^{2}=9.078, p=0.0026, \beta_{\text {time }}=0.473,95 \% \mathrm{CI}=\right.$ $[0.208,0.769])$. We found a similar pattern of results for V1/LOC $\left(\chi_{(1)}^{2}=9.301, p=0.0023, \beta_{\text {time }}=0.456,95 \% \mathrm{CI}=[0.166\right.$, $0.720])$, for V1/parietal $\left(\chi_{(1)}^{2}=7.254, p=0.0071, \beta_{\text {time }}=0.409\right.$; $95 \% \mathrm{CI}=[0.078,0.723])$, for V2/LOC $\left(\chi_{(1)}^{2}=6.775, p=0.0092\right.$, $\left.\beta_{\text {time }}=0.388 ; 95 \% \mathrm{CI}=[0.073,0.701]\right)$, and modestly for V2/ parietal $\left(\chi_{(1)}^{2}=4.293, p=0.038, \beta_{\text {time }}=0.304 ; 95 \% \mathrm{CI}=[0.024\right.$, $0.580])$. We also analyzed changes in the connectivity pattern for LOC/parietal but did not find evidence of reliable changes over time $\left(\chi_{(1)}^{2}=1.01, p=0.3151, \beta_{\text {time }}=0.141 ; 95 \% \mathrm{CI}=[-0.152\right.$, $0.407])$. We found similar null results when analyzing changes in the connectivity pattern between $\mathrm{V} 1$ and precentral (i.e., motor cortex $)\left(\chi_{(1)}^{2}=1.294, p=0.255, \beta_{\text {time }}=0.156,95 \% \mathrm{CI}=\right.$ $[-0.112,0.413]), \mathrm{V} 2$ and precentral $\left(\chi_{(1)}^{2}=1.541, p=0.214\right.$, $\left.\beta_{\text {time }}=0.164,95 \% \mathrm{CI}=[-0.092,0.446]\right)$, LOC and precentral $\left(\chi_{(1)}^{2}=0.166, p=0.683, \beta_{\text {time }}=0.055 ; 95 \% \mathrm{CI}=[-0.253\right.$, $0.337])$, and parietal and precentral $\left(\chi_{(1)}^{2}=0.257, p=0.612\right.$, $\left.\beta_{\text {time }}=0.069 ; 95 \% \mathrm{CI}=[-0.201,0.354]\right)$.

Overall, these results suggest that repeated drawing practice may lead to enhanced transmission of object-specific information between regions in occipital and parietal cortex over time (i.e., from early to late in production phase; Fig. 6).

\section{Enhanced object evidence not found in activation patterns within regions}

The foregoing connectivity analyses were based on voxels from pairs of ROIs. Thus, it may have been the case that simply combining information about voxel activity from two regions would have been sufficient to uncover learning effects that were masked when regions were considered individually. If true, then concatenating voxel activation patterns from two ROIs should also reveal changes in target information over time. To test this possibility directly, we constructed the same type of classifier on the concatenated voxel activation patterns extracted from each ROI, rather than their connectivity patterns.

By contrast with decoding from connectivity patterns, we found that, when using concatenated activation patterns from V1 and V2, including time as a predictor did not improve model fit $\left(\chi_{(1)}^{2}=0.075, p=0.784\right)$, and time did not predict target selection $\left(\beta_{\text {time }}=-0.030,95 \% \mathrm{CI}=[-0.257,0.181]\right)$. There were similarly null effects for concatenated V1/LOC $\left(\chi_{(1)}^{2}=0.690, p=\right.$ $\left.0.406, \beta_{\text {time }}=0.092,95 \% \mathrm{CI}=-0.126,0.302\right), \mathrm{V} 1 /$ parietal $\left(\chi_{(1)}^{2}=\right.$ $\left.0.203, p=0.652, \beta_{\text {time }}=-0.054,95 \% \mathrm{CI}=[-0.315,0.180]\right)$, V2/LOC $\left(\chi_{(1)}^{2}=0.274, p=0.601, \beta_{\text {time }}=0.059,95 \% \mathrm{CI}=\right.$ 

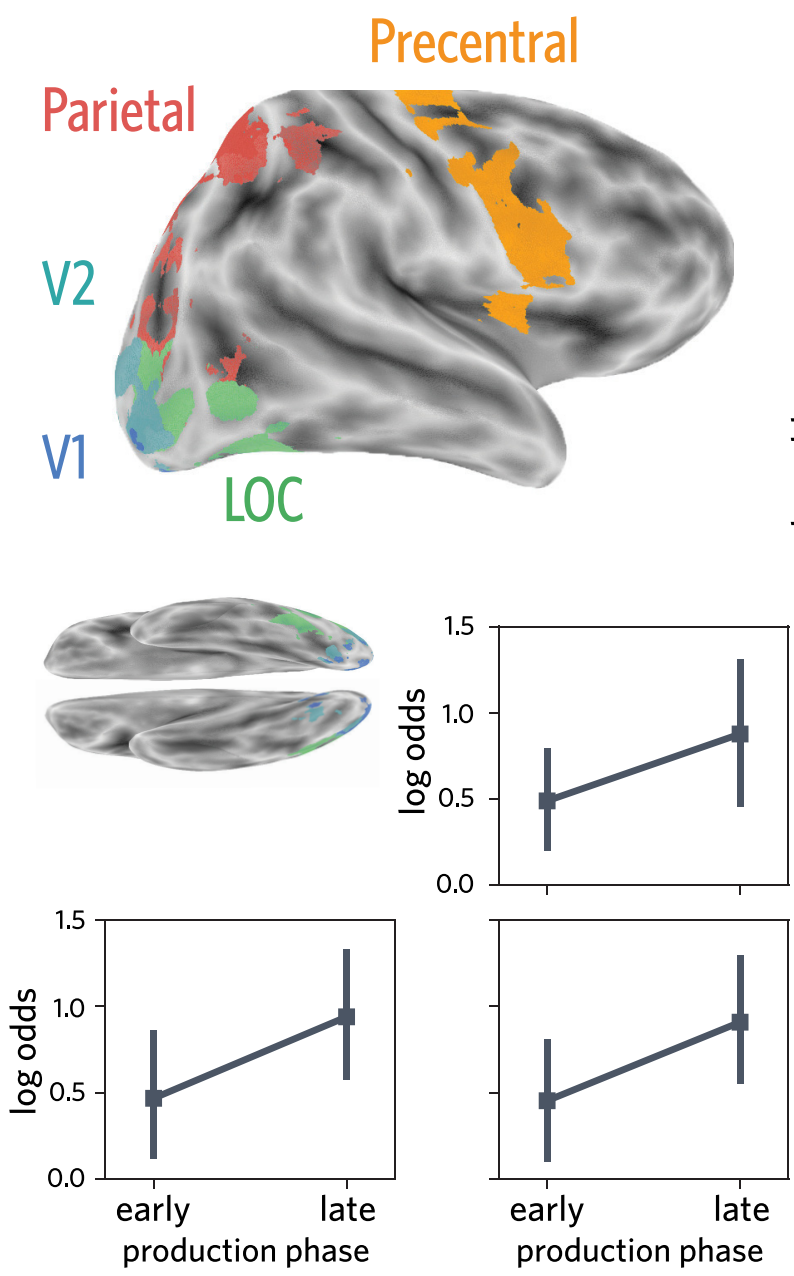

V2
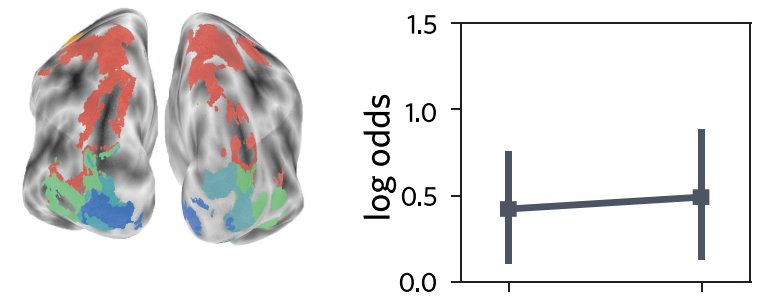

\section{Parietal}
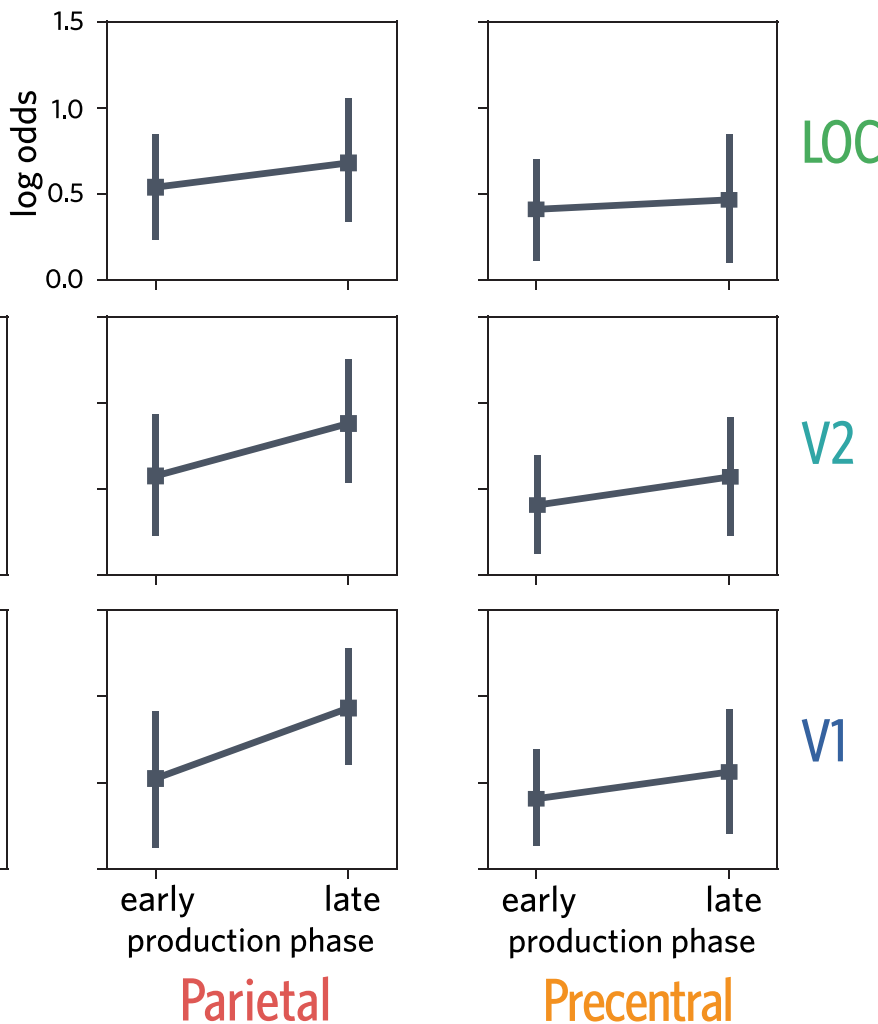

Figure 6. Target selection over time during production, trained on connectivity patterns between pairs of regions. Target selection measured using connectivity patterns in production-related voxels in V1, V2, LOC, parietal cortex, and precentral gyrus. Error bars indicate $95 \% \mathrm{Cls}$.

$[-0.171,0.273]), \mathrm{V} 2 /$ parietal $\left(\chi_{(1)}^{2}=0.301, p=0.583, \beta_{\text {time }}=\right.$ $-0.066,95 \% \mathrm{CI}=[-0.315,0.158])$, and LOC/parietal $\left(\chi_{(1)}^{2}=\right.$ $\left.0.000, p=0.988, \beta_{\text {time }}=-0.002,95 \% \mathrm{CI}=[-0.246,0.251]\right)$.

Together, these results suggest that connectivity patterns between regions carry task-related information about the target object that was not redundant with information directly accessible from activation patterns within regions. A possibility consistent with these findings is that enhanced target information in patterns of connectivity between occipitotemporal and parietal regions may reflect increasing ability to emphasize the diagnostic features of an object across repeated attempts to transform their perceptual representation of the object into an effective motor plan to draw it. This interpretation would be consistent with prior studies using a similar paradigm that have shown improvement in the recognizability of drawings across repetitions (Fan et al., 2018; Hawkins et al., 2019). More broadly, our analyses present a general approach to quantifying how multivariate patterns of connectivity between regions change during repeated practice of complex visually guided actions, including visual production (Vinci-Booher et al., 2016).

\section{Discussion}

The current study investigated the functional relationship between recognition and production of objects in human visual cortex. Moreover, we aimed to characterize the consequences of repeated production on the discriminability of object representations. To this end, we scanned participants using fMRI while they performed both recognition and production of the same set of objects. During the production task, they repeatedly produced drawings of two objects. During the recognition task, they repeatedly discriminated the repeatedly drawn objects, as well as a pair of other control objects. We measured spatial patterns of voxel activations in ventral visual stream during drawing production and found that regions in occipital cortex carried diagnostic information about the identity of the currently drawn object that was similar in format to the pattern evoked during visual recognition of a realistic rendering of that object. Moreover, we found that these production-related activation patterns reflected sustained prioritization of the currently drawn object in visual cortex and concurrent suppression of the other repeatedly drawn object, suggesting that visual production recruits an internal representation of the current object to be drawn that emphasizes its diagnostic features. Finally, we found that patterns of functional connectivity between voxels in occipital cortex and parietal cortex supported progressively better decoding of the currently drawn object across the production phase, suggesting a potential neural substrate for production-related learning. Together, these 
findings contribute to our understanding of the neural mechanisms underlying complex behaviors that require the engagement of, and interaction between, regions supporting perception and action in the brain.

Our findings advance an emerging literature on the neural correlates of visually cued drawing behavior. The studies that comprise this literature have used widely varying protocols for cueing and collecting drawing data. For example, one early study briefly presented watercolor images of objects as visual cues, and instructed participants to use their right index finger, which lay by their side and out of view, to "draw" the object in the air (Makuuchi et al., 2003). Another study used diagram images of faces and had participants produce their drawings on a paperbased drawing pad, also hidden from view (Miall et al., 2009). More recently, however, MR-compatible digital tablets have enabled researchers to automatically capture natural drawing behavior in a digital format while participants are concurrently scanned using fMRI. In one such study, participants copied geometric patterns (i.e., spiral, zigzag, serpentine), which were then projected onto a separate digital display (Yuan and Brown, 2014), while another had participants copy line drawings of basic objects, but participants were unable to view the strokes they had created (Planton et al., 2017).

Unlike the way people produce drawings in everyday life, participants in these studies generally did not receive visual feedback about the perceptual properties of their drawing while producing it (compare Yuan and Brown, 2014), and were cued to produce simple abstract shapes rather than real-world objects. By contrast, in the current study, we used photorealistic renderings of $3 \mathrm{D}$ objects as visual cues and gave participants continuous visual access to their drawing while producing it. Using photorealistic object stimuli rather than geometric patterns or preexisting line drawings of objects allowed us to interrogate the functional relationship between the perceptual representations formed during visual recognition of real-world objects and those that are recruited online to facilitate drawing production. Moreover, participants in our study received immediate visual feedback about the perceptual properties of their drawing while producing it, allowing us to investigate distinctive aspects of drawing behavior that are not shared with other depictive actions (e.g., gesture) that do not leave persistent visible traces.

Previous studies were also primarily concerned with characterizing overall differences in BOLD signal amplitude between a visually cued drawing and another baseline visual task (i.e., object naming, subtraction of two visually presented numbers). The current study diverges from prior work in its use of machine learning techniques to analyze the expression of object-diagnostic information within visual cortex, as well as in the pattern of connections to downstream parietal regions. As a consequence, our study helps to elucidate the neural content and circuitry that underlie visual production behavior.

The current findings are generally consistent with prior work in observing broad recruitment of a network of regions during visually guided drawing production, including regions in the ventral stream and in parietal cortex. Moreover, our findings are congruent with a growing body of evidence showing a large degree of functional overlap in the network of regions during the perception and production of abstract symbols (James and Gauthier, 2006; James, 2017), as well as overlap between regions recruited during production of symbols and object drawings (Planton et al., 2017). This convergence suggests that common functional principles (Lake et al., 2015), if not identical neural mechanisms, may underlie fluent perception and production of symbols and object drawings, in particular the recruitment of representations in visual cortex and computations in parietal cortex that are thought to transform perceptual representations into actions (Vinci-Booher et al., 2019).

Interestingly, the most robust information about which object participants were currently drawing was available in occipital cortex. These results are largely consistent with prior work that has found functional overlap between neural representations of perceptual information and information in visual working memory (Harrison and Tong, 2009; Sprague et al., 2014) and visual imagery (Kosslyn et al., 2001; Dijkstra et al., 2017). Thus, a natural implication for our understanding of the neural mechanisms underlying visual production is that mechanisms supporting visual working memory and visual imagery in sensory cortex are also recruited during production of a drawing of an object held in working memory. Further, these mechanisms may have provided the basis for our ability to decode the identity of the target object during drawing production.

A potential alternative explanation for above-chance decoding of object identity in occipital cortex could be that participants made consistent eye movements in response to the object when presented as a cue in the production runs or as an image in the recognition runs. However, this explanation seems unlikely for a few reasons: First, objects were presented briefly and centrally during recognition runs, reducing the need and time available for object-specific eye movements. Second, objects were displayed from a trial-unique viewpoint during both recognition and production runs, ensuring variation in the retinal input across repeated presentations of the same object and, thus, the patterns of eye movements made in response. Third, participants were instructed to draw the object as it was displayed in the cue during production runs, which meant that their drawings and associated eye movements also reflected viewpoint variability.

While the current study was focused on learning-related consequences of visual production practice, other learning studies that have used different tasks, including categorization training (Jiang et al., 2007), associative memory retrieval (Favila et al., 2016), spatial route learning (Chanales et al., 2017), and statistical learning (Schapiro et al., 2012), have found that repeated engagement with similar items can lead to their differentiation in the brain. Although we did not find that trained object representations became more differentiated within our ROIs, we discovered in exploratory analyses that the pattern of connectivity between occipital and parietal regions during drawing production carried increasingly diagnostic information about the target object across the production phase. Our current findings suggest that, while activation patterns evoked by objects within subregions of occipital cortex may not differentiate as a result of repeated production, the manner in which this information is transmitted between occipital and parietal regions might. Further investigation of how perceptual information represented in visual cortex is transformed into motor commands issued by downstream regions, including motor cortex, is a clear and important direction for future research (Churchland et al., 2012; Russo et al., 2018). It is plausible that some visual properties may map selectively onto specific motor plans such that otherwise similar stimuli may lead to increasingly different actions as participants learn to emphasize the visual properties of an object that distinguish it from other objects in their drawings. We did not find evidence for such changes in linear transformations between visual and motor cortex, but future work could examine nonlinear transformations (Anzellotti and Coutanche, 2018) or better ways to 
characterize motor representations and learning (Berlot et al., 2018).

Together, our findings provide support for the notion that visual production and recognition recruit functionally similar representations in human visual cortex, and that learning may occur during visual production by enhancing the discriminability of neural representations of repeatedly practiced items (Wang et al., 2015). In the long run, further application of multivariate analysis approaches to neural data collected during visual production may shed new light not only on the representation of task-relevant information in sensory cortex, but also how this information is transmitted to downstream parietal and frontal regions that support the planning and execution of complex motor plans (Goodale and Milner, 1992; James, 2017).

\section{References}

Anzellotti S, Coutanche MN (2018) Beyond functional connectivity: investigating networks of multivariate representations. Trends Cogn Sci $22: 258-269$.

Bainbridge WA, Hall EH, Baker CI (2019) Drawings of real-world scenes during free recall reveal detailed object and spatial information in memory. Nat Commun 10:5.

Bates D, Maechler M, Bolker B, Walker S, Christensen RH, Singmann H, Ropp L (2015) Package 'lme4.' Convergence, 12:1.

Berlot E, Popp NJ, Diedrichsen J (2018) In search of the engram, 2017. Curr Opin Behav Sci 20:56-60.

Chanales AJ, Oza A, Favila SE, Kuhl BA (2017) Overlap among spatial memories triggers repulsion of hippocampal representations. Curr Biol 27: 2307-2317.e5.

Chun MM, Golomb JD, Turk-Browne NB (2011) A taxonomy of external and internal attention. Annu Rev Psychol 62:73-101.

Churchland MM, Cunningham JP, Kaufman MT, Foster JD, Nuyujukian P, Ryu SI, Shenoy KV (2012) Neural population dynamics during reaching. Nature 487:51-56.

Cichy RM, Chen Y, Haynes JD (2011) Encoding the identity and location of objects in human loc. Neuroimage 54:2297-2307.

Cohen JD, Daw N, Engelhardt B, Hasson U, Li K, Niv Y, Norman KA, Pillow J, Ramadge PJ, Turk-Browne NB, Willke TL (2017) Computational approaches to fMRI analysis. Nat Neurosci 20:304-313.

Cumming G (2014) The new statistics: why and how. Psychol Sci 25:7-29.

Davachi L (2006) Item, context and relational episodic encoding in humans. Curr Opin Neurobiol 16:693-700.

Dijkstra N, Bosch SE, van Gerven MA (2017) Vividness of visual imagery depends on the neural overlap with perception in visual areas. J Neurosci 37:1367-1373.

Draschkow D, Wolfe JM, Võ ML (2014) Seek and you shall remember: scene semantics interact with visual search to build better memories. J Vis 14:10.

Efron B, Tibshirani RJ (1994) An introduction to the bootstrap. Boca Raton, FL: CRC.

Epstein R, Graham KS, Downing PE (2003) Specific scene representations in human parahippocampal cortex. Neuron 37:865-876.

Fan JE, Turk-Browne NB (2013) Internal attention to features in visual short-term memory guides object learning. Cognition 129:2.

Fan JE, Yamins DL, Turk-Browne NB (2018) Common object representations for visual production and recognition. Cogn Sci 42:2670-2698.

Favila SE, Chanales AJ, Kuhl BA (2016) Experience-dependent hippocampal pattern differentiation prevents interference during subsequent learning. Nat Commun 7:11066.

Garvert MM, Dolan RJ, Behrens TE (2017) A map of abstract relational knowledge in the human hippocampal-entorhinal cortex. Elife 6:e17086.

Gazzaley A, Nobre AC (2012) Top-down modulation: bridging selective attention and working memory. Trends Cogn Sci 16:129-135.

Gegenfurtner KR, Kiper DC, Fenstemaker SB (1996) Processing of color, form, and motion in macaque area v2. Vis Neurosci 13:161-172.

Goodale MA, Milner AD (1992) Separate visual pathways for perception and action. Trends Neurosci 15:20-25.

Grill-Spector K, Kourtzi Z, Kanwisher N (2001) The lateral occipital complex and its role in object recognition. Vision Res 41:1409-1422.

Gross CG (1992) Representation of visual stimuli in inferior temporal cortex. Philos Trans R Soc Lond B Biol Sci 335:3-10.
Güclü U, van Gerven MA (2015) Deep neural networks reveal a gradient in the complexity of neural representations across the ventral stream. J Neurosci 35:10005-10014.

Harrison SA, Tong F (2009) Decoding reveals the contents of visual working memory in early visual areas. Nature 458:632-635.

Hawkins R, Sano M, Goodman N, Fan J (2019) Disentangling contributions of visual information and interaction history in the formation of graphical conventions. In: Proceedings of the 41st Annual Conference of the Cognitive Science Society. Montreal, Canada.

Haxby JV, Gobbini MI, Furey ML, Ishai A, Schouten JL, Pietrini P (2001) Distributed and overlapping representations of faces and objects in ventral temporal cortex. Science 293:2425-2430.

Hubel DH, Wiesel TN (1968) Receptive fields and functional architecture of monkey striate cortex. J Physiol 195:215-243.

Hung CP, Kreiman G, Poggio T, DiCarlo JJ (2005) Fast readout of object identity from macaque inferior temporal cortex. Science 310:863866

James KH (2017) The importance of handwriting experience on the development of the literate brain. Curr Direct Psychol Sci 26:502-508.

James KH, Atwood TP (2009) The role of sensorimotor learning in the perception of letter-like forms: tracking the causes of neural specialization for letters. Cogn Neuropsychol 26:91-110.

James KH, Gauthier I (2006) Letter processing automatically recruits a sensory-motor brain network. Neuropsychologia 44:2937-2949.

Jiang X, Bradley E, Rini RA, Zeffiro T, Vanmeter J, Riesenhuber M (2007) Categorization training results in shape-and category-selective human neural plasticity. Neuron 53:891-903.

Kamitani Y, Tong F (2005) Decoding the visual and subjective contents of the human brain. Nat Neurosci 8:679-685.

Kosslyn SM, Ganis G, Thompson WL (2001) Neural foundations of imagery. Nat Rev Neurosci 2:635-642.

Kourtzi Z, Kanwisher N (2001) Representation of perceived object shape by the human lateral occipital complex. Science 293:1506-1509.

Lake BM, Salakhutdinov R, Tenenbaum JB (2015) Human-level concept learning through probabilistic program induction. Science 350:13321338.

Lewis-Peacock JA, Postle BR (2012) Decoding the internal focus of attention. Neuropsychologia 50:470-478.

Li JX, James KH (2016) Handwriting generates variable visual output to facilitate symbol learning. J Exp Psychol Gen 145:298-313.

Longcamp M, Boucard C, Gilhodes JC, Anton JL, Roth M, Nazarian B, Velay JL (2008) Learning through hand-or typewriting influences visual recognition of new graphic shapes: behavioral and functional imaging evidence. J Cogn Neurosci 20:802-815.

Makuuchi M, Kaminaga T, Sugishita M (2003) Both parietal lobes are involved in drawing: a functional MRI study and implications for constructional apraxia. Cogn Brain Res 16:338-347.

Miall RC, Gowen E, Tchalenko J (2009) Drawing cartoon faces: a functional imaging study of the cognitive neuroscience of drawing. Cortex 45:394406.

Murray EA, Bussey TJ (1999) Perceptual-mnemonic functions of the perirhinal cortex. Trends Cogn Sci 3:142-151.

Norman KA, Polyn SM, Detre GJ, Haxby JV (2006) Beyond mind-reading: multi-voxel pattern analysis of fMRI data. Trends Cogn Sci 10: $424-430$.

Planton S, Longcamp M, Péran P, Démonet JF, Jucla M (2017) How specialized are writing-specific brain regions? An fMRI study of writing, drawing and oral spelling. Cortex 88:66-80.

Russo AA, Bittner SR, Perkins SM, Seely JS, London BM, Lara AH, Miri A, Marshall NJ, Kohn A, Jessell TM, Abbott LF, Cunningham JP, Churchland MM (2018) Motor cortex embeds muscle-like commands in an untangled population response. Neuron 97:953-966.e8.

Rust NC, DiCarlo JJ (2010) Selectivity and tolerance ("invariance") both increase as visual information propagates from cortical area $\mathrm{v} 4$ to it. J Neurosci 30:12978-12995

Sayim B, Cavanagh P (2011) What line drawings reveal about the visual brain. Front Hum Neurosci 5:118.

Schapiro AC, Kustner LV, Turk-Browne NB (2012) Shaping of object representations in the human medial temporal lobe based on temporal regularities. Curr Biol 22:1622-1627.

Serences JT, Ester EF, Vogel EK, Awh E (2009) Stimulus-specific delay activity in human primary visual cortex. Psychol Sci 20:207-214. 
Sprague TC, Ester EF, Serences JT (2014) Reconstructions of information in visual spatial working memory degrade with memory load. Curr Biol 24:2174-2180.

Tipper SP, Weaver B, Houghton G (1994) Behavioural goals determine inhibitory mechanisms of selective attention. Q J Exp Psychol A 47:809-840.

Vinci-Booher S, James TW, James KH (2016) Visual-motor functional connectivity in preschool children emerges after handwriting experience. Trends Neurosci Educ 5:107-120.

Vinci-Booher S, Cheng H, James KH (2019) An analysis of the brain systems involved with producing letters by hand. J Cogn Neurosci $31: 138-154$.

Walther DB, Chai B, Caddigan E, Beck DM, Fei-Fei L (2011) Simple line drawings suffice for functional MRI decoding of natural scene categories. Proc Natl Acad Sci U S A 108:9661-9666.

Wammes JD, Meade ME, Fernandes MA (2016) The drawing effect: evidence for reliable and robust memory benefits in free recall. Q J Exp Psychol 69:1752-1776.

Wang Y, Cohen JD, Li K, Turk-Browne NB (2015) Full correlation matrix analysis (FCMA): an unbiased method for task-related functional connectivity. J Neurosci Methods 251:108-119.

Yamins DL, Hong H, Cadieu CF, Solomon EA, Seibert D, DiCarlo JJ (2014) Performance-optimized hierarchical models predict neural responses in higher visual cortex. Proc Natl Acad Sci U S A 111:8619-8624.

Yuan Y, Brown S (2014) The neural basis of mark making: a functional MRI study of drawing. PLoS One 9:e108628. 